\title{
Claves del éxito para la viralización de contenidos de salud. El caso de las redes sociales del Hospital Povisa
}

\section{Keys to success for the viralization of health content. Social media study of the Povisa Hospital}

\author{
Alba López-Bolás; Mónica Valderrama-Santomé; Francesca Di-Virgilio
}

Cómo citar este artículo:

López-Bolás, Alba; Valderrama-Santomé, Mónica; Di-Virgilio, Francesca (2019). "Claves del éxito para la viralización de contenidos de salud. El caso de las redes sociales del Hospital Povisa". El profesional de la información, v. 28, n. 5, e280502.

https://doi.org/10.3145/epi.2019.sep.02

Artículo recibido el 31-12-2018

Aceptación definitiva: 10-05-2019

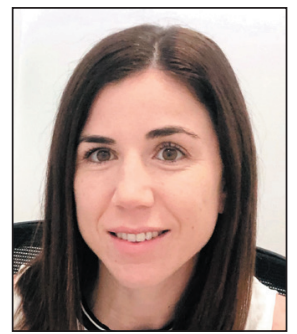

Alba López-Bolás $\bowtie$

https://orcid.org/0000-0002-3885-4007

Universidad de Santiago de Compostela Facultad de Ciencias de la Comunicación Avda. de Castelao, s/n. Campus Norte. 15782 Santiago de Compostela (La Coruña), España alba.lopez.bolas@usc.es

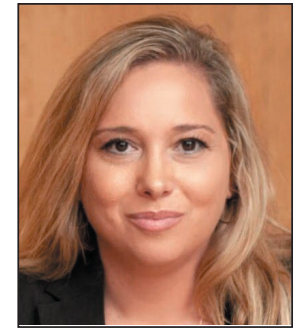

Mónica Valderrama-Santomé https://orcid.org/0000-0003-2299-3659

Universidad de Vigo

Edificio Miralles. Campus universitario.

36310 Vigo (Pontevedra), España

santome@uvigo.es

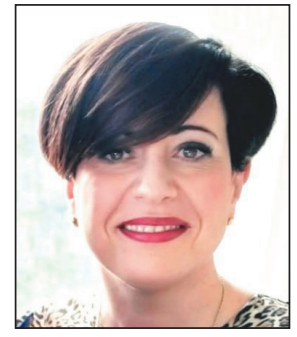

\section{Francesca Di-Virgilio}

https://orcid.org/0000-0001-6017-9506

Università degli Studi del Molise

Dipartimento di Economia

Via F. De Sanctis. 86100 Campobasso, Italia

fradivi@unimol.it

\section{Resumen}

Cuando la información se comparte se consume de forma más reflexiva y resulta más sencillo comprenderla y asimilarla. Esto provoca que la participación de la audiencia social esté entre las principales preocupaciones de un comunicador del sector sanitario. Para esta investigación se han analizado las interacciones generadas en el perfil de Facebook del hospital privado más grande de España, el Hospital Povisa, entre 2016 y 2018. Los resultados demuestran que los contenidos que más se comparten son los que transmiten emociones positivas, con imágenes de pacientes y profesionales sanitarios, con frases directas y afirmativas procedentes de fuentes de información propias, y contados a través de historias reales vividas por pacientes. Las conclusiones alcanzadas pueden servir como fórmulas eficaces de conexión entre otros hospitales y sus pacientes actuales y potenciales.

\section{Palabras clave}

Comunicación sanitaria; Comunicación corporativa; Comunicación organizacional; Redes sociales; Medios sociales; Hospitales; Información sanitaria; Comunicación hospitalaria; Viralización; Facebook; Hospital Povisa.

\footnotetext{
Abstract

When information is shared it is consumed in a more reflective manner and it is easier to understand and assimilate it, so seeking the participation of the social audience should be among the main concerns of a health sector communicator. The study performs an analysis of the interactions generated in Facebook of the largest private hospital in Spain, the Povisa Hospital, between 2016 and 2018. The results show that the posts that are most shared are those that transmit
} 
positive emotions, with images of patients and health professionals, with direct and affirmative phrases, coming from our own sources of information, and told through real stories lived by patients. The conclusions reached in this study can serve as effective formulas of connection between other hospitals and their social audience.

\section{Keywords}

Health communication; Organizational communication; Corporate communication; Social networks; Social media; Hospitals; Health information; Hospital communication; Viralization; Facebook; Povisa Hospital.

\section{Introducción}

Transmitir información es insuficiente para comunicar con éxito en una organización. Es necesaria una correcta gestión del contenido informativo y una adaptación a los medios que ofrece internet. En el sector sanitario la gestión de la reputación en internet ha adquirido importancia en los últimos años. Los cambios económicos, sociales y políticos, y el impacto cada vez mayor de las redes sociales en el ámbito empresarial e institucional han provocado reacciones de todo tipo por parte de las organizaciones hospitalarias.

En la actualidad definimos las redes sociales como los sitios web que actúan como plataformas participativas y autoexpresivas (Schultz, 2007) gestionadas por empresas u organizaciones que permiten que los individuos construyan y mantengan relaciones con sus contactos o amigos a través de la creación de perfiles de usuario y de forma rápida y flexible, mediante el uso de la tecnología (Martorell-Castellano, 2014).

Un cambio fundamental producido por el auge de los canales online es la necesidad de investigar y conocer continuamente los mensajes que se difunden en las comunidades virtuales sobre la entidad y sobre el sector en el que está inmersa (García-Orosa, 2009). Existen nuevos emisores y el departamento de comunicación debe conocerlos bien. Además, el uso de nuevas herramientas en internet se ha vuelto clave para dar respuesta a lo que actualmente se espera de los hospitales, como organizaciones encargadas no sólo de la asistencia médica y la calidad asistencial del paciente sino también de la formación de la población en los hábitos de vida saludables.

Como apunta Martorell-Castellano (2014), la participación en una comunidad de marca virtual se define como el grado en el que un individuo se involucra en sus actividades e interactúa con el resto de miembros. Esta participación es la que va a garantizar el mantenimiento de la comunidad a largo plazo y la que ayudará a la organización a cumplir sus objetivos comunicativos. Para que una estrategia de comunicación online hospitalaria funcione debe tener en cuenta a los usuarios compartiendo sus contenidos. De hecho, la comunicación unidireccional a audiencias masivas está desapareciendo en un entorno de medios emergentes en el que el alcance de la información no es simplemente la suma de personas expuestas, sino que debe tener en cuenta la probabilidad de que el receptor vuelva a transmitir la información a través de alguna plataforma para otras personas (Cappella; Kim; Albarracín, 2015).

La importancia de analizar las características que provocan la viralización de contenidos en el sector sanitario para ofrecer un mejor servicio, unida a la ausencia de publicaciones académicas que analicen la función de las redes sociales en los hospitales, son las razones que han motivado la elaboración de este trabajo con la intención de que los resultados sean de utilidad tanto en el entorno académico como en el profesional.

\section{Redes sociales en el sector sanitario}

El camino hacia un hospital más humano es el trayecto irrenunciable de toda estrategia de comunicación hospitalaria (Costa-Sánchez, 2012). Para convertir al usuario en eje de la organización, no sólo a nivel teórico sino también en la práctica del día a día, es imprescindible crear un plan de comunicación que incluya los canales que actualmente permiten una mayor relación entre organizaciones y usuarios, como son las redes sociales. Di-Virgilio, Valderrama-Santomé y López-Bolás (2018) también señalan que uno de los grandes desafíos en la implementación de las redes sociales en las organizaciones es la implementación de estructuras organizativas y procesos que sean lo suficientemente flexibles para satisfacer las nuevas demandas de comunicación.

Humanizar implica relación, contacto, consideración del usuario como persona, y requiere escuchar, dialogar y comunicar(se). Las redes sociales permiten cumplir por tanto con uno de los objetivos fundamentales de un hospital, lograr que los usuarios lo identifiquen como su centro de referencia por su prestigio y calidad.

Para medir el grado de satisfacción de los usuarios con el hospital se deben estudiar aspectos de la comunicación que impliquen una participación activa por parte de la comunidad. Varios estudios han demostrado que la información se consume de forma más profunda y reflexiva cuando se comparte. Esta respuesta fue marcada, por ejemplo, por el $73 \%$ de los encuestados del estudio de The New York Times (2011) titulado The psychology of sharing: Why do people share online?

Para medir el grado de satisfacción de la comunidad con un hospital se deben estudiar aspectos de la comunicación que impliquen una participación activa 
Orihuela (2012) también sitúa la apuesta por la comunidad como estrategia eficaz frente a la apuesta por el tráfico, ofreciendo contenidos de calidad que puedan ser compartidos y poniendo la interactividad por encima del número de páginas o contenidos vistos. Así mismo, Dafonte-Gómez (2018) concluye en su estudio sobre los motivos por los que se comparten los contenidos, Motivations and emotions in news sharing, que sin el apoyo de los usuarios cualquier modelo de negocio, formato innovador o nueva vía para la participación en los medios de comunicación está destinada al fracaso, y que los aspectos emocionales desempeñan un papel importante en el proceso de toma de decisiones en cuanto al intercambio de contenidos en internet.

Noguera-Vivo (2015) también analiza el valor de la comunidad en internet bajo tres reglas tácticas del entorno online:

- valor de las conexiones;

- experiencia de la comunidad; $y$,

- la que se utiliza como fundamental en esta investigación, la recomendación social.

Tal y como señala Noguera-Vivo (2015), estudiar qué hace que un contenido sea viral debería estar entre las principales preocupaciones de cualquier comunicador. Esto no significa que lo más compartido sea el principal baremo de calidad, pero los contenidos de calidad deben estar preparados para que se puedan compartir fácilmente.

Además de la calidad, existen otras vías necesarias para provocar la participación ciudadana. Entre ellas, la innovación, factor clave en un entorno tan saturado de información, y la transmisión de ideas positivas y de esperanza, que adquiere aún más protagonismo en el sector sanitario. De hecho, Berger y Milkman (2012) analizaron cerca de 7.000 artículos de The New York Times y observaron que los que transmitían esperanza, inspiración o positivismo fueron compartidos con más frecuencia que aquellos que producían tristeza o negatividad.

La utilización de nuevos medios online puede ayudar a los hospitales a establecer niveles de confianza con sus usuarios comparables a niveles que actualmente disfrutan los propios profesionales sanitarios (Huang; Bolchini; Jones, 2011). Deben plantearse las redes sociales como canal de comunicación y marketing de los servicios del hospital y al mismo tiempo como una oportunidad de formación de pacientes y de proyección de la responsabilidad social (González-Pacanowski, 2012). En cualquier caso, todos los estudios previos analizados coinciden en situar al paciente como eje sobre el que giran los objetivos del plan de comunicación, tanto en el sector privado como en el público.

Las redes sociales de un hospital deben plantearse como una oportunidad de formación de pacientes y proyección de la responsabilidad social

En cuanto a los mensajes lanzados por las organizaciones que forman parte del sector de la salud deben ser más informativos que persuasivos (Del-Río-Pérez; Medina-Aguerrebere, 2012). Esto coincide con la idea de que la información hospitalaria tenga un objetivo de educación al paciente y de enseñanza de los hábitos de vida saludables.

En relación con los públicos principales de las organizaciones hospitalarias, Del-Río-Pérez y Medina-Aguerrebere (2012) hablan del paciente y del profesional de la salud. Costa-Sánchez (2012) va más allá y distingue tres categorías:

- públicos externos: usuarios potenciales del hospital que residen en el área sanitaria;

- internos: profesionales que trabajan en el hospital, estudiantes en formación, entre otros;

- intermedios: usuarios que requieren hacer uso de los servicios del hospital, pacientes, familiares y acompañantes.

Todos ellos están conectados entre sí y deben ser prioritarios en la estrategia comunicativa para crear mensajes coherentes, eficaces y virales.

Las principales características de la comunicación viral son (Welker, 2002):

- gratuidad del contenido o servicio que se distribuye;

- sencillez con la que puede ser redistribuido por un usuario;

- apelación a los intereses de la audiencia para compartir;

- uso de redes de distribución existentes para la transmisión del contenido;

- uso de recursos ajenos para la distribución del mensaje.

Por lo tanto, partimos de la base de que un contenido viral tiene que diseñarse con la intención de que aporte algún beneficio al receptor pero también pensando en el beneficio que se obtiene al compartirlo.

Varias investigaciones mencionadas previamente han demostrado que compartir es una acción que nos ayuda a entender mejor la información que consumimos. Cuando el consumo es social existe un proceso mayor, también colectivo, de reflexión y análisis (Noguera-Vivo, 2015). Por ello y por la importancia que para cualquier marca supone conseguir prescriptores espontáneos que compartan con su comunidad cualquier información generada previamente por la organización, situamos esta acción como la de más valor en las redes sociales del Hospital Povisa, el objeto de estudio de esta investigación.

Otros estudios, como los de Valdis Krebs (Fernández, 2004) también se basan en que la importancia de la posición en un determinado grupo viene determinada por la capacidad de transmitir información al resto del grupo y por la capacidad de servir de enlace entre los diferentes miembros. De acuerdo con lo que señala García-Orosa (2009), el gabinete 
de comunicación, más que preocuparse por una difusión masiva del mensaje, puede utilizar la estrategia de dirigirse a aquellos nodos con mayor influencia en el púbico que le interesa, es decir, implementar una estrategia en dos niveles para que el mensaje se transmita de una forma más rápida y eficaz (la información no llega a través del departamento de comunicación sino a través de amigos del destinatario final).

La gran pregunta es, por tanto, ¿cómo debe ser un contenido en salud para que se comparta? Se parte de la base de que, por demostraciones de estudios ya mencionados, en primer lugar tiene que ser de calidad, con intensidad emocional y con valor diferencial suficiente como para destacar entre multitud de informaciones que inundan las redes sociales cada día, pero ¿cuál es el formato más compartido y la temática que más éxito tiene entre la audiencia? ¿El tema, la fuente de información, el tono y la modalidad del discurso influyen a la hora de provocar la viralización de contenidos?

\section{Objetivos y organización metodológica}

El estudio se centra en analizar los resultados del análisis cuantitativo y cualitativo propio realizado sobre una muestra de contenidos publicados en Facebook por el Hospital Povisa durante dos años.

Povisa es el hospital privado más grande de España y pertenece al grupo gallego Nosa Terra XXI. Está situado en Vigo y cuenta con 40.000 metros cuadrados y 1.500 trabajadores, según datos recogidos en su propia web (2018). El Hospital Povisa ha contado durante 20 años, desde 1999, con un gabinete de comunicación que ha ido incorporando los medios y recursos necesarios para dar respuesta a lo que actualmente exige una correcta gestión de la comunicación, tanto convencional como online.

Con la llegada de internet, tal y como señalaba García-Orosa (2009), los gabinetes no sólo pueden transmitir o gestionar información, sino que además deben realizar una comunicación activa dentro del espacio virtual. La elección de canales e instrumentos implica una determinada filosofía y política de comunicación por parte de los responsables de gestionar la imagen de Povisa.

Con la apertura de su web y sus perfiles en redes sociales a partir de 2012, el hospital deja de tener los medios tradicionales como única vía de comunicación y pone en marcha un nuevo plan de comunicación que le posiciona como uno de los centros hospitalarios nacionales con mayor interacción en internet. Durante este tiempo se ha consolidado también como el hospital gallego con mayor reputación, según los datos del Monitor de Reputación Sanitaria (Merco, 2017). En concreto, ocupa el puesto decimosexto en el ranking 2017 de hospitales privados de España. La metodología de este ranking analiza múltiples fuentes de opinión, como usuarios, asociaciones de consumidores y pacientes, periodistas especializados, profesores universitarios, influencers y sindicatos.

La gestión de las redes sociales por parte del gabinete de comunicación de Povisa, en coordinación con el equipo directivo del hospital, ha permitido que todas las comunicaciones se realicen de forma inmediata, lo que da una mayor transparencia a la organización y amplía el alcance de las noticias y reportajes publicados en medios de comunicación locales y autonómicos, así como en revistas especializadas del sector sanitario.

Para esta investigación se ha seleccionado Facebook por ser el servicio de red social con más usuarios en todo el mundo (2.200 millones de usuarios activos en enero de 2018), según los datos proporcionados por la empresa, y la red social que cuenta con mayor número de seguidores por parte del Hospital Povisa-datos recogidos de la propia red social en noviembre de 2018-.

Se han planteado los siguientes objetivos generales (OG) y específicos (OE):

OG. Determinar los factores que han contribuido en la viralización de contenidos en las redes sociales del hospital entre 2016 y 2018.

OE1. Identificar el tipo de contenido en Facebook que genera más engagement rate.

OE2. Identificar las características y temáticas de las imágenes con mayor engagement rate.

OE3. Identificar las fuentes de información que utiliza el hospital para elaborar los contenidos con mayor engagement rate.

OE4. Identificar el tipo de discurso y la temática principal de los contenidos con mayor engagement rate.

OE5. Identificar la modalidad y el tono que se utiliza en los textos que generan más engagement rate.

La medición del engagement (también denominado compromiso, implicación o enganche) ha sido investigada desde muy diferentes perspectivas en los últimos años. La definición del engagement como el grado de compromiso adquirido por un usuario con una marca es una de las más extendidas (Alonso-González, 2015) y las variables más ampliamente utilizadas para medir el nivel de engagement de los usuarios con las redes sociales son el número de Me gusta, Compartir y Comentar (Ballesteros-Herencia, 2019). En esta investigación se ha decidido incluir también como variable el número de clicks en el post al significar una atención directa del usuario en el contenido y ser un indicador importante sobre la implicación de la audiencia.

En cuanto a la fórmula para calcular el engagement rate en Facebook se ha optado por sumar estas cuatro variables (Me gusta, comentarios, compartidos y clicks) y dividirlo entre el número de usuarios que han visto cada uno de los conteni- 
dos. De esta forma calculamos el porcentaje de usuarios que realizan alguna acción en los contenidos publicados sobre el total de gente que los ha visto.

Esta fórmula es la que propone Popat (2012) para definir el término viralidad, aunque en su caso no selecciona el número de clicks como variable, algo que sí tienen en cuenta por ejemplo Oviedo-García et al. (2014) en su cálculo de lo que denominan "ratio de interés".

Para elaborar esta investigación se ha recurrido a fuentes de información primarias, entendidas como cualquier tipo de indagación en la que el investigador analiza la información que él mismo obtiene (Cea-D’Ancona, 1996), mediante la aplicación de una o varias técnicas de obtención de datos. La técnica de obtención de datos utilizada es el análisis de contenido, fundamental para contrastar empíricamente los datos obtenidos de la visualización de textos, imágenes y vídeos. La objetividad, la sistematicidad y la cuantificación de estos contenidos son los rasgos fundamentales de este análisis. Para la interpretación de los datos se ha recurrido a las variables e indicadores, explicados a continuación.

Se han analizado 908 contenidos de la principal red social del hospital, Facebook, que son todos los publicados en su perfil entre 2016 y 2018. Para estudiar la viralización de contenidos en salud y redactar las conclusiones se ha observado el número de veces que se comparte un contenido y se ha calculado el engagement rate, entendido como el compromiso de la audiencia con la organización, en concreto, el porcentaje de audiencia que reaccionó, compartió, hizo click o comentó una publicación de la página. Este número se calcula dividiendo el número de interacciones totales entre el alcance (el número real de personas que ve un post).

En cuanto a las variables utilizadas para identificar y analizar los contenidos que más se comparten y que generan un mayor compromiso de la audiencia se han seleccionado las siguientes:

- alcance: número total de usuarios únicos que ven un contenido;

- interacción: suma de acciones realizadas por los usuarios en el contenido: clicks, compartidos, comentarios y Me gusta o reacciones;

- engagement rate: porcentaje de usuarios que realizaron alguna interacción en el contenido sobre el total de usuarios que lo vieron.

Posteriormente se realiza una interpretación de los datos a través de un análisis cualitativo en profundidad de cada uno de los contenidos. Este estudio se elabora con el fin de identificar los aspectos de las publicaciones en las redes sociales del hospital que provocan una mayor viralización y recomendación por parte de pacientes potenciales y usuarios en general. Estas son las variables del análisis cualitativo:

- tipo de contenido: se diferencia entre fotografía, link, vídeo, infografía o sólo texto;

- tema de la imagen o vídeo: corresponde a los objetos, personas o iconos mostrados para identificar la temática visual principal del contenido;

- fuente de información: procedencia del contenido, que puede corresponder a medios de comunicación, organizaciones, asociaciones, instituciones, profesionales o al propio hospital (fuente de información propia);

- tipo de discurso principal: forma de plantear un determinado asunto en el texto, es decir, la fórmula elegida para transmitir la información a través de la red social;

- temática: asunto o materia principal del discurso desarrollado;

- modalidad del contenido: actitud del emisor ante el contenido que comunica;

- tono general: entendido como carácter o modo particular de la expresión y del estilo de un texto según el asunto que trata o el estado de ánimo que pretende reflejar y se utiliza para clasificar los contenidos en aquellos que transmitan emociones positivas, negativas o neutras;

- discusión dominante: tipo de discurso o debate que generan los usuarios con sus comentarios publicados en cada post;

- prescripción: capacidad de cada contenido de generar prescriptores que publiquen comentarios positivos sobre la organización.

Finalmente, se identifican las principales conclusiones del análisis para cerrar el artículo estudiando posibles aplicaciones de la estrategia llevada a cabo por el Hospital Povisa para otros centros sanitarios u organizaciones. También se concretan las variables que se pueden tomar como referencia para futuras investigaciones sobre las estrategias llevadas a cabo en las redes sociales del sector sanitario.

\section{Resultados}

\subsection{Análisis de contenido en Facebook}

Como se ha dicho, la principal red social del Hospital Povisa de Vigo es Facebook. El perfil del hospital cuenta con 8.759 seguidores -datos de noviembre de 2018-. La comunidad de fans no representa actualmente el alcance potencial de los contenidos de la red social. De hecho, el alcance promedio de cada uno de los contenidos publicados en el perfil de Facebook de Povisa es de 4.676 personas únicas y, gracias a la viralización de contenidos, el 20\% de todos los posts tienen un alcance de visualización que supera el total de fans de la página. Esto quiere decir que el número de seguidores de una página no es indicador del número de personas reales que puede llegar a ver y compartir el contenido. 
Con la publicación de información propia en Facebook, Povisa logra alcanzar una audiencia de más de 20.000 personas en su área de influencia en varios contenidos publicados sobre salud o sobre el propio hospital. Este alcance se logra con un correcto posicionamiento orgánico, pero también con el impulso, en ocasiones, de la publicidad de pago segmentada con el objetivo de llegar sólo a perfiles influyentes. Para no entrar en detalle sobre la inversión necesaria para llegar a un público determinado, esta investigación se centra sobre todo en el engagement rate, es decir, el porcentaje de la audiencia que de verdad interactúa de alguna forma con la publicación, que muestra interés más allá de ver la fotografía, el vídeo o el texto en su muro de la red social.

El número promedio de usuarios que interactúa con una publicación de Povisa en Facebook es de 312 por post, lo que supone 633 interacciones en total por cada contenido ${ }^{1}$. Durante todo el período analizado en esta investigación se registraron 574.744 interacciones en total (Me gusta, compartidos, comentarios y clicks) y se llegó a más de 4 millones de personas, lo que supone un engagement rate promedio de $11,36 \%^{2}$.

\subsection{Tipo de contenido}

Comenzamos comprobando si el tipo de contenido elegido puede provocar un mayor compromiso por parte de la audiencia social. Una vez analizada toda la muestra, se observa como únicamente con la publicación de fotografías, sin link a otra página, se consigue alcanzar el $30 \%$ de engagement rate, llegando a superar el $50 \%$ en algunos posts. En cuanto al promedio, tal y como se observa en el gráfico 1, cuando se opta por publicar enlaces, con objetivo de captar tráfico a la web del hospital o a otros canales externos a la red social, el compromiso de la audiencia cae, con un promedio de 10,2\% durante los años analizados, frente al 14,29\% que registran de media las fotografías.

En cuanto al formato en vídeo, el promedio de engagement rate se sitúa en un 12,88\%, por lo que logra superar en más de dos puntos al porcentaje de interacción de los contenidos que incluyen link. Los vídeos más eficaces son los utilizados para transmitir historias de usuarios de Povisa y para educar al paciente.

\subsection{Tema de la imagen}

Una vez comprobado que las fotografías son el tipo de contenido que mayor interacción de calidad genera, en el gráfico 2 se observa qué tipo de información se muestra en las fotografías que más se comparten por parte de la audiencia del hospital. El resultado es que cuando los pacientes son los protagonistas de las imágenes, los usuarios tienden a compartir más veces la publicación con su comunidad y el compromiso es mayor. En concreto, durante el período de análisis este tipo de imágenes se compartieron 1.742 veces, lo que supone un promedio de 79 veces por contenido y un engagement rate de $14,87 \%$.

Cuando el tema de la imagen se centra en los profesionales sanitarios, también se alcanza un buen promedio, de 59 veces compartido cada post y un engagement rate de $14,72 \%$. Sin embargo, cuando sólo se muestran personas adultas sin relación con el sector sanitario o niños, el promedio se sitúa por debajo de 20 veces compartido cada post y menos de un $11 \%$ de engagement rate, a pesar de que el texto sí que pueda hacer referencia a temas sobre salud.

Tal y como observamos en el gráfico 2, el engagement rate de los contenidos publicados que muestran personas es superior al de los que incluyen objetos, comida, paisajes o ilustraciones.

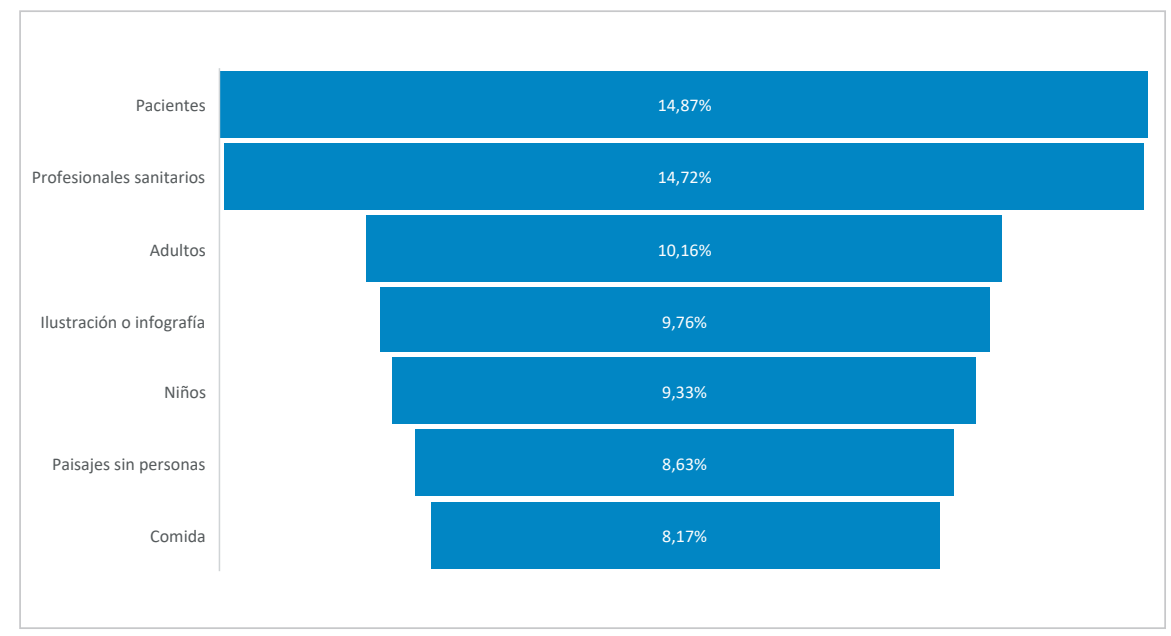

Gráfico 2. Promedio de engagement rate según el tema principal de la imagen 
De hecho, la publicación de ilustraciones (infografías, gráficos, dibujos vectoriales, etc.), sin personas ni objetos reales en la imagen, no provoca participación por parte de la audiencia, registrando sólo un promedio de 22 veces compartido cada post y un $9,76 \%$ de engagement rate.

\section{El engagement rate de los contenidos que muestran pacientes y profesionales sanitarios en la imagen es superior al de los que incluyen objetos, lugares o ilus- traciones}

\subsection{Fuente de información}

Se analiza la procedencia del contenido, que puede corresponder a medios de comunicación, organizaciones, asociaciones, instituciones, profesionales o al propio hospital, y el grado de relación entre la fuente de información y la participación de los usuarios.

Los siete contenidos más compartidos de toda la muestra recogida durante los dos años de análisis, los únicos que superan las 300 veces compartidos, pertenecen a una fuente de información propia del hospital. Esto quiere decir que ha sido generada de forma original por el Gabinete de Comunicación de Povisa en coordinación con el departamento correspondiente del hospital.

El post más compartido en todo el período analizado, con cerca de 500 veces y un engagement rate del 19,68\%, es un contenido con fuente propia, texto sencillo (una única frase afirmativa), imagen con profesional sanitario y tono positivo. Fue visto por 30.360 personas. Además de tratarse de un contenido propio y que transmite emociones positivas en la zona de influencia del hospital, el tema genera gran interés al nombrar al hospital como pionero en preservar la fertilidad en operaciones de cáncer de cuello de útero. Esta situación provoca que la discusión dominante de sus decenas de comentarios publicados sean felicitaciones al médico protagonista de la noticia y recomendaciones del hospital.

Este contenido, junto a la mayor parte de todos los que tienen fuente de información propia y un engagement rate por encima del promedio de la muestra, corresponde a la adaptación de una nota de prensa para ser difundida por redes sociales. Povisa, a lo largo de sus 7 años de presencia online, logra utilizar con éxito sus redes sociales como vía de difusión de notas de prensa. Lo hace tanto con links a su web para ser utilizada por los medios de comunicación, como con la readaptación y publicación del contenido para redes sociales, logrando que su comunidad online más fidelizada le ayude a viralizar la información en un ámbito geográfico y social de referencia para el hospital.

Si ordenamos el contenido teniendo en cuenta la interacción entre el alcance, es decir el compromiso de la audiencia, se observa también que los 10 primeros posts con más engagement rate, superando el $30 \%$, son de fuente propia. Entre ellos se encuentra el que hace referencia al acto de entrega de diplomas de los titulados de Formación Profesional del Centro de Estudios Povisa. Esto se debe a las 18 fotografías que incluye el contenido, acumulando alto índice de interacción en cada una de ellas.

Tal y como se observa en el gráfico 3, los contenidos con los que el hospital consigue una mayor repercusión y compromiso de la audiencia, con un promedio de $13,09 \%$ de engagement rate, proceden de fuente propia, es decir, son posts originales y únicos creados a partir de información que procede del propio hospital. En segundo lugar se sitúan los contenidos procedentes de

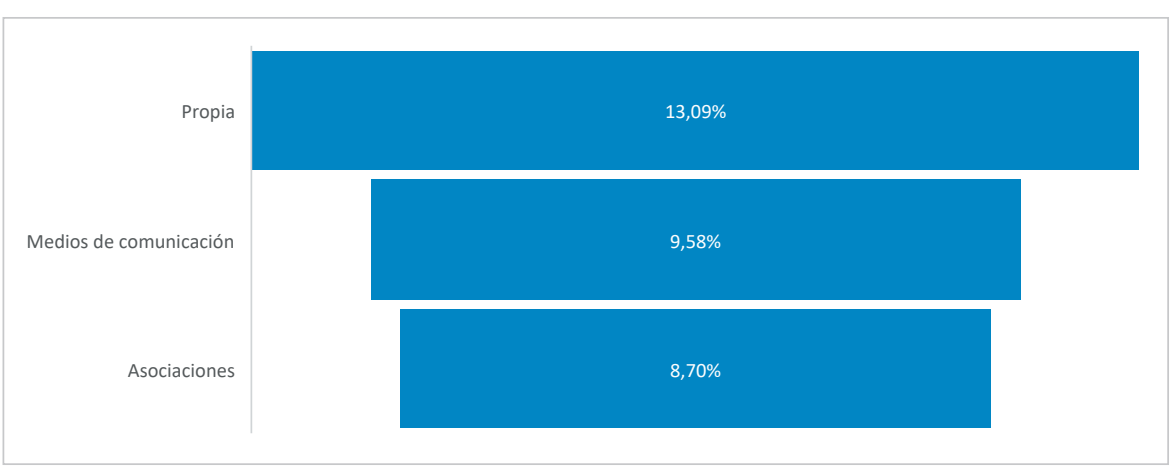

Gráfico 3. Promedio de engagement rate según la fuente de la información

medios de comunicación, con un 9,58\%, siendo Faro de Vigo y La voz de Galicia los que mayor interacción de calidad generan, por encima de medios especializados como Redacción médica, Efe salud o ConSalud.es. Por último se sitúan las asociaciones, una fuente de información recurrida por el hospital para sus contenidos, pero con la que sólo se consigue un promedio de $8,70 \%$.

\subsection{Tipo de discurso y tema principal}

Con el análisis de todos los contenidos publicados en Facebook se demuestra que, al igual que ocurre con el tema de la imagen, situar a los pacientes como protagonistas de la información provoca que sea compartido un mayor número de veces por los usuarios.

Dar visibilidad a través de las redes sociales del hospital a historias reales de pacientes provoca una mayor
Los contenidos de un hospital se comparten más veces cuando los pacientes son los protagonistas de la información que cuando se tratan de forma general consejos o noticias sobre salud 
viralidad e interacción social. Este tipo de contenidos genera emociones variadas y muchos usuarios se sienten identificados, ya sea de forma directa o indirecta por situaciones vividas en su entorno. A través de los comentarios publicados en estos contenidos se detecta una gran empatía por parte de la comunidad de Povisa, que a su vez comparte con su comunidad. Se genera un impacto importante en pacientes potenciales a los que se consigue llegar sin

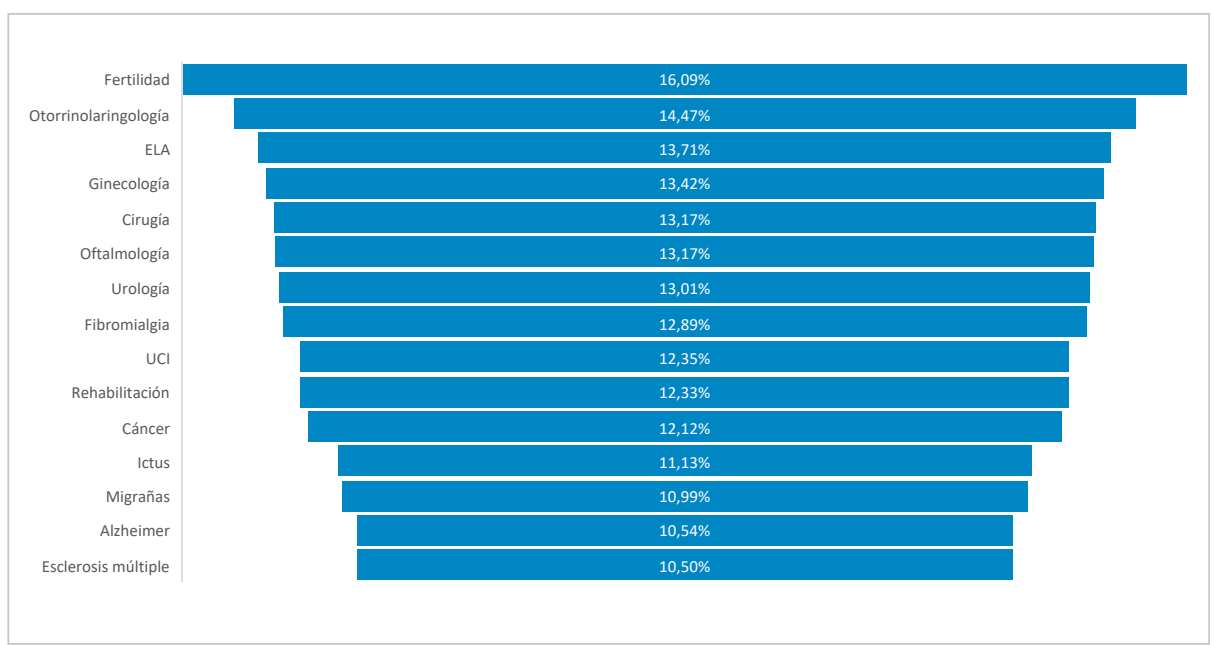

Gráfico 4. Promedio de engagement rate según el tema del contenido ningún tipo de publicidad o mensaje persuasivo, sino simplemente convirtiendo experiencias vividas en contenidos útiles, atractivos y emocionales.

En cuanto a la información generada en el blog con consejos y curiosidades sobre salud, no logran alcanzar un alto grado de interacción de calidad. Es cierto que registran un número alto de clicks en comparación con otro tipo de contenidos y pueden ser utilizados con éxito como captadores de tráfico hacia la web, pero el compromiso de la audiencia no es destacable en estos casos.

Cuando se analiza el asunto concreto del discurso desarrollado (gráfico 4), se demuestra que los contenidos más compartidos y que mayor promedio de engagement rate generan son, por orden, los que tratan temas de fertilidad (16,09\%), otorrinolaringología $(14,47 \%)$, esclerosis lateral amiotrófica (ELA) $(13,71 \%)$, ginecología $(13,42 \%)$, cirugía (13,17\%), oftalmología $(13,17 \%)$ y urología $(13,01 \%)$. Todos los temas mostrados en el gráfico 4 superan además un promedio de 30 veces compartido cada post. La mayoría de estos temas que provocan aumentar la media de interacción de calidad incluyen, como se indicó anteriormente, a pacientes y profesionales sanitarios contando o ilustrando la historia en primera persona.

\subsection{Modalidad y tono general}

Se analiza por un lado la modalidad de cada uno de los contenidos publicados, es decir, la actitud del emisor ante el contenido que comunica y, por otro lado, el tono general de las informaciones, clasificándolas según transmitan emociones positivas, negativas o neutras.

Una vez clasificadas se realiza un análisis comparativo entre los porcentajes de engagement alcanzados y el número de veces que se comparten. El resultado es que las expresiones enunciativas afirmativas simples, que comunican mensajes positivos y optimistas, consiguen que sean compartidas más veces que el resto. Sin embargo, esta interacción cae cuando las frases principales del contenido son exhortativas o interrogativas. Estas últimas, que incluyen preguntas a los usuarios, consiguen respuestas de la audiencia social a sus preguntas, en la mayoría de los casos, pero no destacan entre las más compartidas ni las que generan mayor compromiso de la audiencia, ya que el promedio de engagement rate es sólo un $9,45 \%$.

Cuando se transmiten mensajes negativos, por ejemplo, noticias con datos preocupantes de salud, problemas generales que se producen en la sociedad, comunicados de acontecimientos poco optimistas, etc., los usuarios tampoco generan interacción de valor y el engagement rate se sitúa en 8,7\%.

El compromiso de la audiencia mejora con la utilización reiterada que realiza Povisa de las oraciones positivas y exclamativas, expresando emociones intensas, con las que logra el porcentaje de engagement rate más alto, con un promedio del $14,5 \%$.

\section{Discusión dominante}

La estrategia de Povisa, centrada en la generación de contenido propio para ser difundido tanto en medios digitales y tradicionales como en redes sociales con la intención de provocar interacción y crear prescriptores, se aleja de la gestión de comunicación convencional, en la que la marca es quien emite los mensajes, y éstos se transmiten de forma unidireccional a un público masivo (Martorell-Castellano, 2014). El hospital busca crear una comunidad auténtica con la creación de contenidos originales y variados, orientados a satisfacer las distintas motivaciones de los usuarios, con la participación proactiva de la organización en los perfiles sociales y aprovechando todas las posibilidades que ofrecen estos canales en el entorno hospitalario y en el área de influencia en general. 
Una vez demostrado que los mensajes positivos y que transmiten emociones intensas son los que más valoran la audiencia, es necesario analizar si este tipo de contenidos ayudan también a captar prescriptores que recomiendan el hospital y publican su opinión de forma espontánea.

El papel de los prescriptores de una organización -los usuarios más fieles y que recomiendan el hospital de forma espontánea- es fundamental para lograr la viralización de contenidos y mantener un alto grado de participación social a lo largo del tiempo. De hecho, como apunta García-Orosa (2009), el trabajo colaborativo de diferentes personas que tengan una opinión positiva sobre la entidad es mucho más eficaz en la creación de una imagen positiva que los mensajes remitidos por el propio gabinete de comunicación.

Muchos hospitales tienen una gran comunidad de usuarios fidelizados pero no los estimulan para convertirlos en prescriptores. De poco sirve acumular usuarios satisfechos, si éstos no transmiten su experiencia positiva a otras personas. De ahí la importancia de compartir y recomendar. De hecho, compartir es una de las funciones que Kietzmann et al. (2011) identifican como fundamentales de las redes sociales, definiéndola como la medida en que los usuarios intercambian, distribuyen y reciben contenido. Sugieren además varias implicaciones fundamentales para las organizaciones en este sentido: por un lado la necesidad de evaluar qué puntos de sociabilidad tienen en común sus usuarios e identificar nuevos temas que puedan mediar en sus intereses compartidos. Sin esto, una red social estará formada principalmente por conexiones entre personas, pero sin nada que se conecte entre sí.

Al observar las conversaciones que se generan en los contenidos de mayor viralización de Povisa se demuestra que aunque los comentarios que más publican los usuarios son opiniones generales, éstas no se producen en los contenidos más compartidos. Son las felicitaciones y agradecimientos personales a médicos y profesionales sanitarios los comentarios que más veces publican los usuarios en los contenidos con mayor interacción y engagement rate. Esto demuestra una vez más la relación directa que hay entre la satisfacción de los pacientes y la acción de recomendar y compartir en la red social principal del hospital.

En pocas ocasiones se producen discusiones, debates o conversaciones completas más allá de la propia recomendación y opinión sobre la noticia. Las críticas al hospital o comentarios negativos, que no son eliminados, se producen sobre todo en los contenidos que se refieren explícitamente a campañas publicitarias concretas, como por ejemplo, las que organizó Povisa en los años 2016 y 2017 para darse a conocer como hospital de referencia en determinadas zonas geográficas en donde los pacientes potenciales que todavía no pertenecían a Povisa podían cambiar de hospital.

Los mensajes persuasivos, más propios de la publicidad tradicional, no son los más eficaces en las redes sociales del ámbito hospitalario. Dentro de la estrategia de comunicación que tenía como objetivo principal la captación de pacientes, se logró un resultado mucho más positivo cuando se publicaron en redes sociales historias de pacientes reales y casos concretos vividos dentro del hospital, que cuando se recurría a mensajes directos para sugerir el cambio de hospital.

También es importante destacar que en el $20 \%$ de los contenidos en donde se publicó alguna crítica, se generó un debate dentro de la comunidad, en donde los propios usuarios satisfechos respondían a los comentarios negativos. Esta defensa que se produce sin que el propio hospital intervenga en ningún momento es el resultado de una correcta gestión previa de la comunicación, basada en el reconocimiento y agradecimiento de la participación y en la creación de recompensas indirectas hacia una comunidad fiel mediante la difusión de contenidos útiles y de calidad. De hecho, cuando de forma totalmente espontánea, un paciente actual o potencial, lee una crítica al hospital y responde públicamente defendiéndolo, se ha logrado el mensaje publicitario de mayor valor que puede tener hoy en día el hospital en sus canales sociales.

Involucrar a los profesionales que forman parte de la plantilla de Povisa en la estrategia de comunicación online ayuda a cumplir objetivos comunes que benefician tanto al hospital como a las marcas individuales que corresponden a cada uno de los trabajadores. El compromiso de estos empleados con la marca corporativa es fundamental para lograr esa complementariedad. Esto coincide con la idea de Molares-Cardoso (2015) de que unos empleados bien informados sobre las características de su empresa conforman un canal de comunicación con el exterior muy adecuado que reporta grandes beneficios.

Según Badea (2014), el público externo percibe a los empleados como verdaderos embajadores de la organización y por lo tanto la retroalimentación adquiere un papel importante en la construcción de su reputación. Una de las fórmulas que utiliza Povisa para convertir en propio un contenido publicado sobre el hospital en medios de comunicación es la difusión en su web de la noticia para compartirla posteriormente en redes sociales mencionando al medio. Cuando se envía una nota de prensa, todos los impactos conseguidos en medios online se recopilan y se comparten en un único contenido para redirigir al usuario nuevamente a la página, captar tráfico y mejorar el posicionamiento en internet.

A través de la investigación se demuestra también que las notas de prensa continúan siendo una de las herramientas de comunicación más importantes y habituales en el trabajo diario de los gabinetes de comunicación, independientemente 
de los medios que se utilicen para su difusión. Constituyen un instrumento eficaz para dar a conocer a la opinión pública cualquier acontecimiento que la organización considere relevante para sus fines (Busto-Salinas, 2013).

En cuanto a la utilidad del vídeo en redes sociales para la educación del paciente es una de las fórmulas reveladas como efectivas en la comunicación hospitalaria. El vídeo ya ha sido demostrada como efectivo en el entorno digital en estudios previos a este trabajo, señalándolo como medio que combina "atributos de entretenimiento, emocionales y de marca" y como instrumento efectivo para la educación del paciente en internet (Huang; Bolchini; Jones, 2011).

\section{Limitaciones e investigaciones futuras}

Este trabajo cuenta con la limitación de ser un estudio de caso, el del Hospital Povisa, y por tanto los resultados no son necesariamente representativos del tipo de contenido que genera mayor viralidad en las redes sociales de cualquier organización del sector sanitario. La relevancia de los resultados radica en que Povisa es el hospital privado más grande de España y uno de los que cuenta con mayor reputación y actividad en las redes sociales. Además, durante la investigación y tras realizar entrevistas previas con los responsables de la comunicación del hospital, se ha tenido acceso a datos exclusivos sobre la interacción de los usuarios y la repercusión de los contenidos, a los que sólo se puede acceder de forma interna, como pueden ser el número de clicks que registra un contenido o el alcance real de usuarios únicos que ven los posts. Esto aporta una mayor validez a los resultados del trabajo, al no depender de las estadísticas que nos muestran sistemas de monitorización externos, sino de los propios datos extraídos del perfil de Facebook del hospital durante los dos años que ha durado la investigación.

En cuanto a investigaciones futuras sobre este tema, sería necesario profundizar en las motivaciones y características que impulsan a los usuarios a compartir contenidos en las redes sociales. De acuerdo con Kümpel, Karnowski y Keyling (2015) hay tres motivos principales:

- egoístas: atraer la atención de las personas, entretenerse o escapar de la rutina diaria, etc.;

- altruistas: con la intención de intercambiar información;

- sociales: necesidad de interactuar con otros, socializar, obtener reputación social, etc.

Se podrían destacar además tres áreas principales de investigación sobre los métodos para que las noticias sean compartidas (Dafonte-Gómez, 2018):

- el estudio sobre las características y motivaciones de los usuarios y organizaciones que comparten noticias;

- la investigación sobre la tipología de los contenidos de los artículos más ampliamente compartidos;

- la investigación sobre las características de las plataformas mediante las cuales se comparten esos artículos.

\section{Conclusiones}

Con esta investigación hemos demostrado que los contenidos que más se comparten en la comunidad online de un hospital son los que transmiten emociones positivas, con imágenes de personas (pacientes o profesionales sanitarios), con frases directas y afirmativas, procedentes de fuentes de información propias y con información contada a través de historias reales vividas por pacientes. Este tipo de contenidos son los que generan interacciones en redes sociales de más valor, provocan un conocimiento duradero sobre un hospital y funcionan también como captadores de pacientes potenciales en el futuro al generar prescriptores y recomendaciones espontáneas.

También se ha comprobado que para generar un compromiso de la audiencia a largo plazo, un alto engagement rate, se debe apostar por frases exclamativas y el paciente debe ser siempre el protagonista de la información. Los aspectos emocionales desempeñan un papel clave en el proceso de toma de decisiones en cuanto a difundir o no contenidos en redes sociales. Aunque dentro de la estrategia del hospital se encuentre también la difusión de información útil con consejos y curiosidades sobre temas de salud y hábitos de vida saludables, este tipo de contenidos no producen un alto compromiso de la comunidad y se sitúan por debajo del promedio de engagement rate total.

Por lo general, los usuarios tienden a compartir más veces una publicación cuando detectan que ha sido generada de forma original por el propio hospital, es decir, por su gabinete de comunicación en coordinación con el departamento correspondiente. Las notas de prensa, adaptadas para difundirse en redes sociales, constituyen un instrumento eficaz para crear contenidos virales en salud. Esto ocurre porque incluyen elementos clave para que se compartan fácilmente por usuarios ya fidelizados. Nos referimos por ejemplo al valor diferencial y original de la información y a la inclusión de profesionales sanitarios como verdaderos embajadores de la organización. Además, el feedback que genera este tipo de contenidos informativos y originales es siempre positivo. Los mensajes persuasivos, más propios de la publicidad tradicional, no son los más eficaces en las redes sociales del ámbito hospitalario.
Los contenidos que más se comparten en la comunidad online de un hospital son los que transmiten emociones positivas, con imágenes de personas (pacientes o profesionales sanitarios), con frases directas y afirmativas 
Como se ha demostrado, dar visibilidad a través de las redes sociales del hospital a historias reales de pacientes provoca una mayor viralidad e interacción social. Este tipo de contenidos genera emociones de todo tipo y muchos usuarios se pueden sentir identificados, ya sea de forma directa o indirecta por situaciones vividas en su entorno. Se genera un impacto importante en pacientes potenciales a los que se consigue llegar de forma indirecta, sin necesidad de inversión publicitaria, convirtiendo experiencias vividas en contenidos útiles, atractivos y emocionales.

Con el análisis de las discusiones y conversaciones que se producen en las redes sociales del hospital se demuestra la relación directa que hay entre la viralización de los contenidos y la satisfacción de los pacientes y usuarios en general. En las informaciones que más veces se comparten, los comentarios son felicitaciones y agradecimientos personales a médicos y profesionales sanitarios.

Una de las fórmulas que se presentan como idóneas para medir el grado de efectividad en la gestión de la comunicación hospitalaria tiene que ver con la capacidad de generar prescriptores. Cuando los pacientes actuales y potenciales, tal y como ocurre en Povisa, publican de forma espontánea comentarios positivos e intervienen en discusiones en las que se critica al hospital para defenderlo y recomendarlo, se ha conseguido el objetivo más valioso de una estrategia de comunicación online.

Con este artículo se comprueba cómo, además de la calidad y el valor diferencial, existen otras vías necesarias para provocar la participación ciudadana a través de estrategias en redes sociales. Además, todos los públicos objetivos del hospital, no sólo pacientes y trabajadores actuales, sino también pacientes potenciales, perfiles influyentes, estudiantes y usuarios en general, deben ser prioritarios en el plan de comunicación para crear mensajes coherentes, eficaces y virales.

Por último, teniendo en cuenta los resultados del caso del Hospital Povisa y con el fin de que puedan contribuir a una mejora de la gestión de las redes sociales en otros hospitales y organizaciones sanitarias, recomendamos las siguientes prácticas:

- involucrar a los pacientes, tanto actuales como potenciales, y a los profesionales sanitarios en la estrategia de comunicación digital;

- elaborar contenido propio y original, y recurrir lo menos posible a fuentes externas;

- utilizar textos, imágenes y vídeos emotivos, que transmitan emociones positivas para captar una mayor atención por parte de la audiencia en redes sociales;

- evitar la difusión de noticias negativas sobre salud, y si es necesario difundir este tipo de informaciones hacerlo de forma creativa y original, y dando visibilidad siempre a la parte positiva de cada situación;

- publicar infografías e ilustraciones con moderación y dar prioridad siempre que se pueda a imágenes reales en vez de a dibujos;

- no recurrir nunca a mensajes persuasivos para conseguir objetivos a través de Facebook, el contenido debe respetar al usuario y aportar valor, resolver un problema sobre salud, informar, emocionar o motivar;

- no eliminar comentarios negativos publicados por usuarios en Facebook, sino que el objetivo del gestor de comunicación hospitalaria debe ser construir una comunidad comprometida y satisfecha para que sean los propios prescriptores espontáneos del hospital los que de forma natural respondan a críticas y generen debate en redes sociales;

- utilizar el engagement rate de Facebook como indicador principal para medir el compromiso de la audiencia social de un hospital.

\section{Notas}

1. Un único usuario puede hacer varias interacciones en una publicación, por eso el número promedio de interacciones es mayor que el de usuarios únicos. Se ha sumado el total de interacciones registradas (datos extraídos del propio perfil del hospital en Facebook) y se ha dividido entre el número de publicaciones analizadas (que son los 908 posts publicados durante el período que se indica en la metodología). Es decir 574.744 interacciones dividido entre 908 posts = 633 interacciones por post.

2. El engagement rate se calcula dividiendo la interacción de cada post entre los usuarios que la vieron (alcance). El promedio de estos 908 posts es 11,36\%. O, lo que es lo mismo, dividir las 574.744 interacciones entre el alcance total $x$ 100. En este caso no se especifica el dato exacto del alcance total en el artículo al no considerarlo relevante, pero como se indica es de más de 4 millones.

\section{Referencias}

Alonso-González, Marián (2015). "Las redes sociales como canal de comunicación de las marcas de moda españolas. El caso de Zara, Mango y El Corte Inglés". Index.comunicación, v. 5, n. 1, pp. 77-105.

http://journals.sfu.ca/indexcomunicacion/index.php/indexcomunicacion/article/view/177

Badea, Marius (2014). "Social media and organizational communication". Procedia. Social and behavioral sciences, v. 149, pp. 70-75.

https://doi.org/10.1016/j.sbspro.2014.08.192 
Ballesteros-Herencia, Carlos A. (2019). "El índice de engagement en redes sociales, una medición emergente en la comunicación académica y organizacional”. Razón y palabra, v. 22, n. 3 102, pp. 96-124.

http://www.revistarazonypalabra.org/index.php/ryp/article/view/1261

Berger, Jonah; Milkman, Katherine L. (2012). "What makes online content viral?”. Journal of marketing research, v. 49, n. 2, pp. 192-205.

https://doi.org/10.1509/jmr.10.0353

Busto-Salinas, Lorena (2013). "Análisis estadístico de los aspectos que influyen en la divulgación de las notas de prensa". En: Mendieta-Ramírez, Angélica (coord.). Tendencias de vanguardia en comunicación. Madrid: Visión Libros, p. 14. ISBN: 9788415965848

Cappella, Joseph N.; Kim, Hyun-Suk; Albarracín, Dolores (2015). "Selection and transmission processes for information in the emerging media environment: Psychological motives and message characteristics". Media psychology, v. 18, n. 3, pp. 396-424.

https://doi.org/10.1080/15213269.2014.941112

Cea-D’Ancona, María-Ángeles (1996). Metodología cuantitativa. Estrategias y técnicas de investigación social. Madrid: Síntesis. ISBN: 9788477384205

http://www.trabajosocial.un/p.edu.ar/uploads/docs/metodologia_cuantitativa_estrategias_y_tecnicas_de_investigacion_ social_cea_d_ancona.pdf

Costa-Sánchez, Carmen (2012). “Estrategias de comunicación corporativa”. En: Medina-Aguerrebere, Pablo; González-Pacanowski, Toni. Comunicación hospitalaria: un plan para el siglo XXI. Madrid: Fragua, pp. 107-125. ISBN: 97884 70745232

Dafonte-Gómez, Alberto (2018). "Audience as medium: Motivations and emotions in news sharing". International journal of communication, v. 12, pp. 2133-2152.

http://hdl.handle.net/11093/1062

Del-Río-Pérez, Jorge; Medina-Aguerrebere, Pablo (2012). "El mensaje y la creatividad en la comunicación hospitalaria". En: Medina-Aguerrebere, Pablo; González-Pacanowski, Toni. Comunicación hospitalaria: un plan para el siglo XXI. Madrid: Fragua, pp. 209-225. ISBN: 9788470745232

Di-Virgilio, Francesca; Valderrama-Santomé, Mónica; López-Bolás, Alba (2018). “Social media strategy within organizational communication: major open issues and challenges". En: Cantoni, Franca; Mangia, Gianluigi (eds.). Human resource management and digitalization. Torino: Giappichelli Routledge, pp. 205-225. ISBN: 9781138313354

Fernández, Sonia (2004). Dos grados: Networking 3.0. Madrid: Lid Editorial Empresarial. ISBN: 8488717644

García-Orosa, Berta (2009). Gabinetes de comunicación on line. Claves para generar información corporativa en la red. Sevilla: Comunicación Social. ISBN: 9788496082786

González-Pacanowski, Toni (2012). “Medios transgénicos para hospitales mutantes”. En: Medina-Aguerrebere, Pablo; González-Pacanowski, Toni. Comunicación hospitalaria: un plan para el siglo XXI. Madrid: Fragua, pp. 193-208. ISBN: 978 8470745232

Huang, Edgar; Bolchini, Davide; Jones, Josette F. (2011). “Users' consumption of healthcare videos on hospital web sites". International journal of pharmaceutical and healthcare marketing, v. 5, n. 1, pp. 43-53.

https://doi.org/10.1108/17506121111121578

Kietzmann, Jan H.; Hermkens, Kristopher; McCarthy, Ian P.; Silvestre, Bruno S. (2011). "Social media? Get serious! Understanding the functional building blocks of social media". Business horizons, v. 54, n. 3, pp. 241-251.

https://doi.org/10.1016/j.bushor.2011.01.005

Kümpel, Anna-Sophie; Karnowski, Veronika; Keyling, Till (2015). “News sharing in social media: A review of current research on news sharing users, content, and networks". Social media + society, v. 1, n. 2.

https://doi.org/10.1177/2056305115610141

Martorell-Castellano, Cristina (2014). “iHazte fan! Las comunidades de marca en Facebook”. En: Fernández-Quijada, David; Ramos-Serrano, Marina (coords.). Tecnologías de la persuasión. Uso de las TIC en publicidad y relaciones públicas. Barcelona: Editorial UOC, p. 120. ISBN: 9788490642030

Merco (2017). Monitor empresarial de reputación corporativa.

http://merco.info/es/monitor-reputacion-sanitaria-hospitales?edicion=2017

Molares-Cardoso, Julinda (2015). "La necesidad de profesionales que gestionen su personal branding frente a la desaparición de los profesionales commodity”. En: Díaz-Fouces, Óscar; García-Soidán, Pilar. Redes y retos. Estudios sobre la comunicación en la era digital. Barcelona: Editorial Octaedro, pp. 119-138. ISBN: 9788499218007 
Noguera-Vivo, José-Manuel (2015). Todos, todo. Manual de periodismo, participación y tecnología. Barcelona: Editorial UOC. ISBN: 9788491160380

Orihuela, José-Luis (2012). 80 claves sobre el futuro del periodismo. Madrid: Grupo Anaya. ISBN: 9788441529823

Oviedo-García, María-Ángeles; Muñoz-Expósito, Miriam; Castellanos-Verdugo, Mario; Sancho-Mejías, María (2014). "Metric proposal for customer engagement in Facebook". Journal of research in interactive marketing, v. 8, n. 4, pp. 327-344.

https://doi.org/10.1108/JRIM-05-2014-0028

Popat, Vee (2012). "What is the typical virality rate for Facebook posts?". VPDM digital marketing, Nov. 30.

https://www.vpdm.ca/typical-virality-rate-for-facebook-posts

Schultz, Don-Edward (2007). "Social call". Marketing management, v. 16, n. 4, pp. 9-11.

The New York Times (2011). The psychology of sharing: Why do people share online?. The New York Times Customer Insight Group.

https://www.bostonwebdesigners.net/wp-content/uploads/POS_PUBLIC0819-1.pdf

Welker, Carl B. (2002). "The paradigm of viral communication”. Information services \& use, v. 22, n. 1, pp. 3-8. https://www.learntechlib.org/p/95985

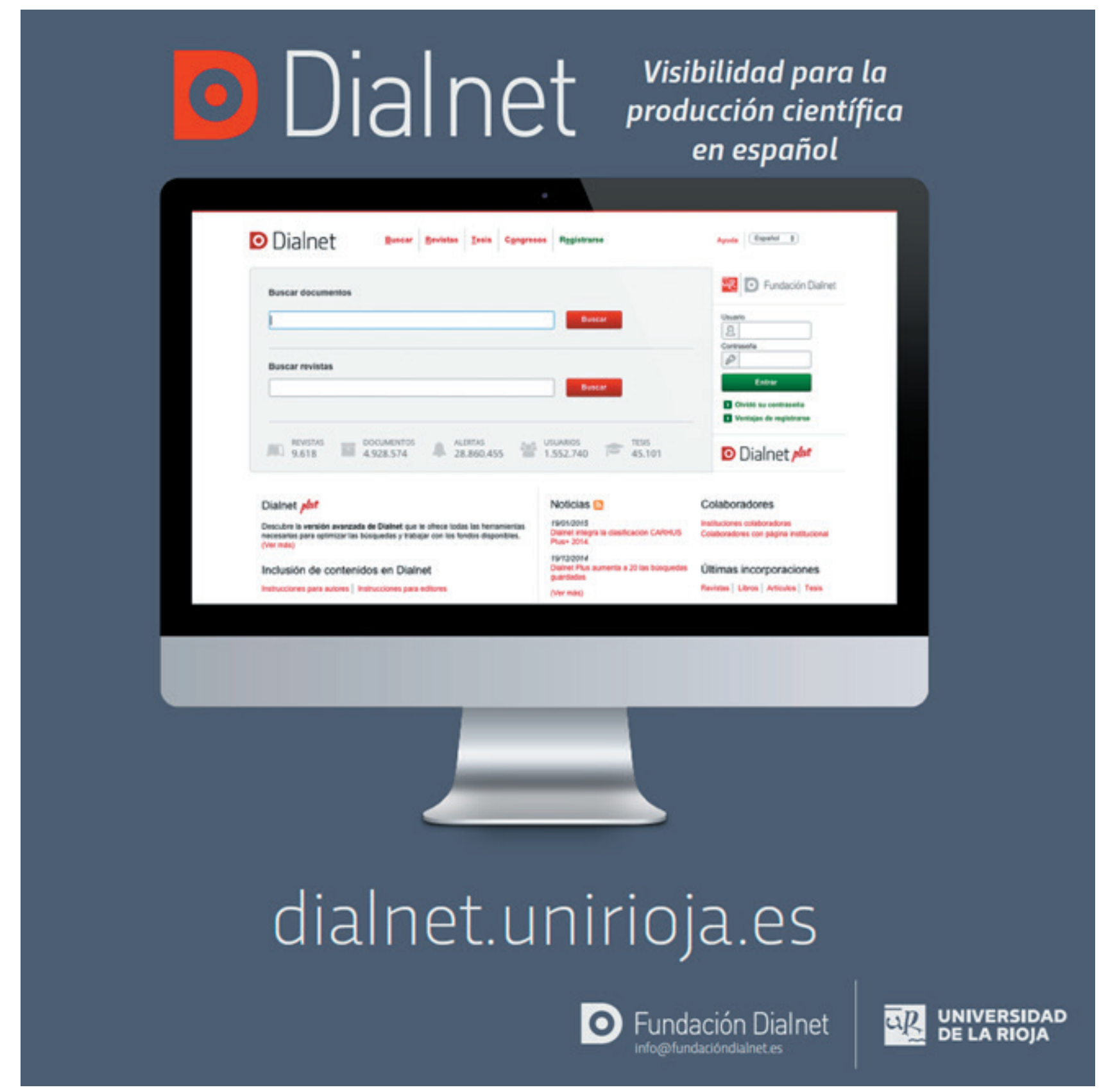

